\title{
OVERVIEW OF DIGITAL FORENSICS ALGORITHMS IN DSLR CAMERAS
}

\author{
E. Aminova ${ }^{\text {a }}$ I. Trapeznikova ${ }^{\mathrm{a}}$ A. Priorov ${ }^{\mathrm{a}}$ \\ a Yaroslavl State University, Yaroslavl, Russian Federation - lena@piclab.ru
}

Commission II, WG II/5

KEY WORDS: DSLR cameras, digital image forensics, fixed pattern noise, hot pixel, flat field

\begin{abstract}
:
The widespread usage of the mobile technologies and the improvement of the digital photo devices getting has led to more frequent cases of falsification of images including in the judicial practice. Consequently, the actual task for up-to-date digital image processing tools is the development of algorithms for determining the source and model of the DSLR (Digital Single Lens Reflex) camera and improve image formation algorithms. Most research in this area based on the mention that the extraction of unique sensor trace of DSLR camera could be possible on the certain stage of the imaging process into the camera. It is considered that the study focuses on the problem of determination of unique feature of DSLR cameras based on optical subsystem artifacts and sensor noises.
\end{abstract}

\section{INTRODUCTION}

The problem of creating of DSLR camera noise pattern has some practical applications in several vectors of human daily life. There are several areas of science and technology, which use standard methods of getting noise from the image, appearing at different stages of image formation system. Firstly, the filtering of medical images, for which the presence of noise is critical, often not a decisive ordinary linear (median) filtering methods. Secondly, for images provided from astronomical systems for the same reasons. Finally, digital images taken from DSLR cameras can be used as eevidence base in forensic examination. In the images of the mentioned above types, the size of the object often coincides with the size of the mask of the used filter of the smallest size, can be lost due to the use of ordinary filtering.

\subsection{Image resolution}

Resolution is perhaps the most important parameter for the selection of the camera. Images must be able to record the fine detail revealed by the microscope magnification. Digital images are made up of millions of tiny squares called picture elements or pixels. These tiny pixels are used to display or print images and the more pixels in a given area the higher the resolution of the image. If a digital image is enlarged there will come a point when the individual elements can be seen as separate dots - similar to graining in a silver halide photograph and the more pixels an image contains the more it can be enlarged before the separate pixels start to show. The size of the image can be described by its dimensions, for example, $1500 \times 1700$ pixels or by the total number of pixels present, in this case, 2.55 million. Resolution is also often quoted as the size of charged coupled device (CCD), which is effectively the number of pixels on the chip. It should be noted, however, that the size of the individual pixels varies amongst different types of CCDs. For instance, for microscopy, a pixel size (square) of 6.7 micrometers is thought to be ideal.

As light enters the camera it passes a filter that divides the pixels into red, green and blue tone pixels - the colors used create the overall color image. The light rays are then directed to the CCD, which is specialized semiconductor that transforms the light rays into electrical charges. The intensity of the electrical charges is proportional to the intensity of the light coming from the subject.
Values stored in the digital image specify the brightness and color of each pixel.

Therefore, the task of adaptive these image processing algorithms is an actual.

\subsection{Noise reduction in medical images}

It is known that for a single CT (computer tomography) scan, the dose of irradiation may exceed the annual dose received from natural sources. Of course, it is possible to reduce the dose of radiation during a medical examination, but this will lead to an increase in noise in the final image, close to Gaussian white noise (Kijewski, 1987). Consequently, the problem of developing algorithms for filtering these noise is important, which would preserve objects that are important for medical examination in images (Reiter, 2006 and Thinh, 2012). To solve this problem, two Rank-2.5D and NLM-2.5D algorithms are known (Strozhilova, 2012). These approaches are algorithms for adaptation to the processing of three-dimensional images of nonlocal mean (Buades, 2005) and rank-filtering algorithms. Since there are a lot of different algorithms that depend on a set of parameters, in parallel with the task of creating effective filtering algorithms, the problem of comparing them with each other and assessing the quality of their work is actual. Traditional quality measurement metrics such as PSNR (Peak-to-Signal Noise Ratio) and MSE (Mean Square Error) do not cope with the task, since they do not distinguish between small variations in brightness and contrast in the image as a whole and small noise and artefacts such as the disappearance of small but significant objects or the shift of the boundary between objects by several pixels in the local area of the image. It should be noted, that if the false positives errors can still occur, then the false negatives errors for medical images are critical.

Simple methods for suppressing noise on medical images, such as linear filtering, together with true noise, suppress highfrequency image the objects of interest that appear on the difference frame, representing changes on the filtered image during the operation of the filtering algorithm in one iteration.

It is known that the noise in a CT image has a Gaussian contrast distribution and a low dispersion level, while objects of interest 
in the image have a higher dispersion value and a more compact distribution in the image. This is a correlation approach to assessing the quality of the filtering algorithms. Another approach is based on the concept of information entropy as a measure of disorder. Consequently, it can be assumed that if the difference image has a small amplitude, then the entropy will also have a small value of magnitude. Along with the increase in the noise reduction power, the brightness of the difference frame will also increase. The brightness value of the image will increase, and the entropy will grow at the same time. However, as soon as the erroneously filtered objects and fragments of the border regions of objects begin to appear on the difference image, the number of regions with approximately zero brightness will increase in the images of the directed differences, and the entropy will decrease. Thus, the value of the maximum entropy is close to the values of the subjective evaluation of the operation of noise cancellation algorithms. Nevertheless, one of the disadvantages of the entropy approach is the excess of the maximum of the surrounding values, while the correlation method can differ by several times.

\subsection{DSLR camera calibration}

The high cost of professional-level DSLR cameras is often caused by the use of Complex calibration algorithms before obtaining a scene image in device by manufacturers such as Canon, Nikon, etc. Consequently, the actual task is to develop efficient, computationally less expensive and inexpensive compared to foreign methods of calibration of image getting devices, but not inferior in quality.

\subsection{Digital image forensics}

Due to the rapid development of user programs in the field of image processing and computer graphics processing, problems regarding the verification of digital images become topical. In addition, these issues are even more acute in the field of forensic examination of the image validation. The methods of criminalistics of digital images are aimed at solving these problems by using the properties of the images themselves. When deciding on the authenticity and admissibility as evidence of a digital image, there are three related important problems. First, this is the method of obtaining the image in question. Most of the researches in this area is focused on detecting computer generated objects in the image and post processing artefacts. Another key problem in the forensic examination of images is the identification and analysis of image features that are relevant to the recording device.

Consequently, in these areas of development of science and technology, the problem of searching for the traces of unique noises features of an image-forming device and calculating its amplitude with the purpose of developing a correct algorithm for its filtering that does not affect the data is actual.

\section{IMAGE FORMATION IN DIGATAL CAMERAS}

The most research in this area based on the mention that the extraction of unique noise trace of DSLR camera could be possible on the certain stage of the imaging process into the camera.

The first approach in this area tries to detect the source cameramodel of the image by determining the differences in image formation pipeline, e.g., processing techniques and component technologies. For this purpose, (Kharrazi,2004) utilized a set of image features which includes color characteristics, image quality metrics, and wavelet coefficient statistics, (Choi, 2006) considered optical distortions due to a type of lens and incorporated it with the features of (Kharrazi,2004), and (Swaminathan, 2007) and (Long, 2006) explored the differences due to choice of color filter array and the corresponding demosaicing algorithm. The main difficulty with camera-model identification is that many camera-models and brands use components by a few manufacturers, and processing methods remain same or very similar among different models of a brand. Hence, reliable identification of class characteristics of a camera requires consideration of many different factors.

The basic architecture and sequence of processing steps remain very similar in all digital cameras (despite the proprietary nature of the underlying technology). The basic structure of image formation pipeline in digital cameras can be illustrated as given in Figure 2.

In a digital camera, the light entering the camera through the lens is first filtered (the most important being an anti-aliasing filter) and focused onto an array of charge-coupled device (CCD) elements, i.e., pixels. The CCD array is the main and most expensive component of a digital camera. Each light sensing element of CCD array integrates the incident light over the whole spectrum and obtains an electric signal representation of the scenery. Since each CCD element is essentially monochromatic, capturing color images requires separate CCD arrays for each color component. However, due to cost considerations, in most digital cameras, only a single CCD array is used by arranging them in a pattern where each element has a different spectral filter, typically one of red, green or blue (RGB). This mask in front of the sensor and it is called the color filter array (CFA). Consequently, each CCD element only senses one band of wavelengths, and the raw image collected from the array is a mosaic of red, green and blue pixels. Since human visual system is more sensitive to green light, CFA patterns typically have more green values than red and blue. The most popular kinds of CFA are represented in Figure 1.

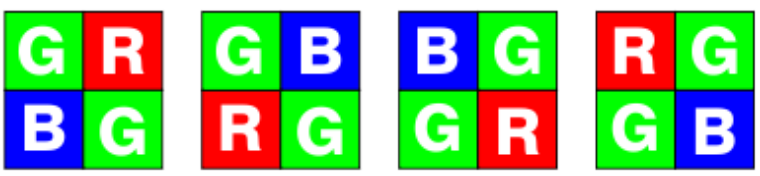

Figure 1. The used types of CFA in RGB color space

\section{DSLR CAMERA UNIQUE FEATURES}

The most of up-to-date digital forensics algorithms based on special features of digital camera, which are strongly connected with traces of a few types of DSLR camera noises.

It should be noted that in order to solve the problems indicated in the relevance of this work, it is necessary to confine oneself to those types of noise and distortion that are available for observation and detection, that is, they can be obtained technically (experimentally) with measurement of the parameters of the distortions and noise obtained, or observed by subjective assessment of experts. Moreover, the influence of other types of noise not considered can be neglected due to their insignificant contribution to the final noise component in the image.

\subsection{Optical traces}

Optical defects can occurs the several sensors of a digital device, because of which the smallest significant data on the final image can be lost. This type of feature, which can be used for digital 


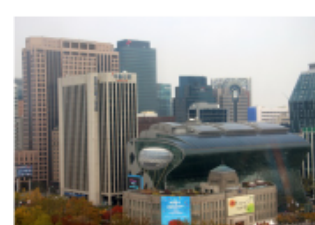

The scene

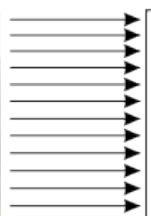

Optical

filters
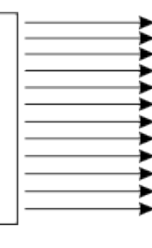

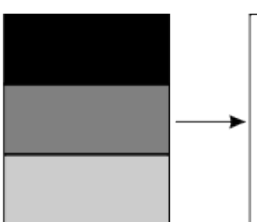

Sensors with CFA

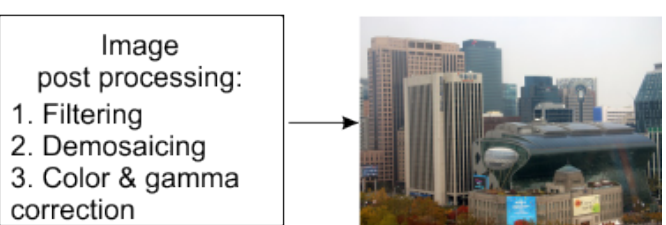

Digital image

Figure 2. Image formation pipeline

forensic, could be divided on two-mail group: one is the defects of construction made during the manufacturing and another one the artefacts caused by a various external factors and foreign objects on the DSLR camera lens.

\subsubsection{Design distortions}

If the lens are brought closer to the lamp (almost closely), even inside the new lens it is possible see dust, villi, bubbles, etc. Usually a small amount of dust and tiny bubbles is acceptable. In practice, the presence of scratches and bubbles on the front lens practically does not affect the image quality, but it can have a psychological effect, especially when buying expensive optics. However, scratches and bubbles on the back lens it is a negative characteristic of camera lens quality. It should be mentioned that than the defects closer to the matrix, the more impact they have on the final image.

Also during the camera's special tests the back-focus and front- focus could be depicted in capturing image as an autofocus subsystem artefacts of DSLR camera.

Chromatic aberrations are most pronounced in zoom lenses and clearly appear on strongly contrasting objects, for example, around tree branches against the background of a bright sky. This is a lateral chromatic aberration. There are a longitudinal chromatic aberration. One simple way to evaluate them is to make a macro of a white sheet of paper with black text at an angle.

And, finally, it is necessary to take into account the optical characteristics of each camera: sharpness and its uniformity over the frame field, as well as to evaluate distortion and vignetting.

\subsubsection{Dusts}

Digital single lens reflex cameras suffer from a well-known sensor dust problem due to interchangeable lenses that they deploy (Dirik, 2007). The dust particles that settle in front of the imaging sensor create a persistent pattern in all captured images.

Distortions of this type include various external factors, such as dust particles on the lens, dirt, traces of water droplets, etc. The instance of this type of artifacts for camera, which was used in experiments, is represented in Figure 4.

A novel source camera identification method based on detection and matching of these dust-spot characteristics was proposed. Dust spots in the image are detected based on a (Gaussian) intensity loss model and shape properties. The model of the spot could be described as:

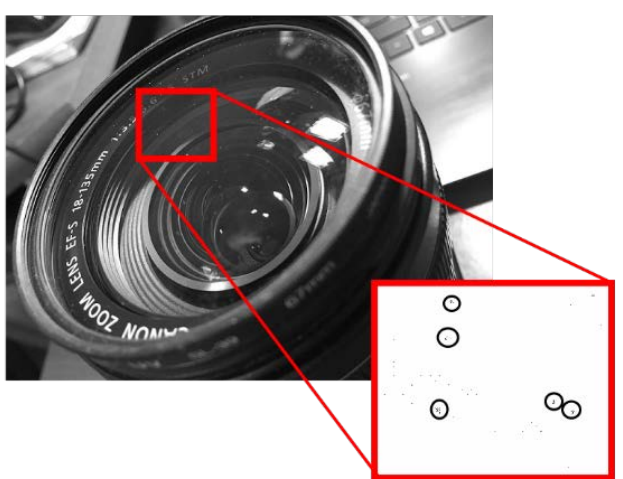

Figure 4. Optical artefacts on camera lens of Canon 700D

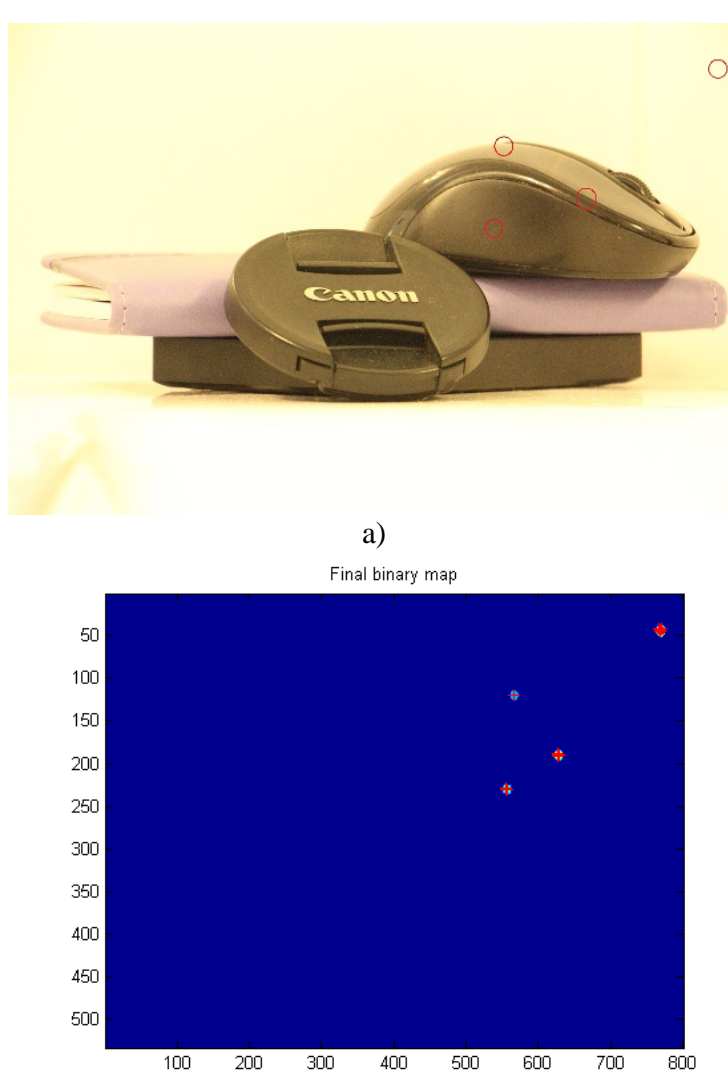

b)

Figure 5. Results of dust pattern constriction for Canon 700D: a) dust pattern for real image taken from camera; b) dust pattern 


$$
\begin{aligned}
\text { intensity } \operatorname{loss}(x, y) & =\frac{-G}{\sqrt{2 \pi \sigma^{2}}} \cdot e^{-(x-\omega)^{2}+\frac{(\omega-y)^{2}}{2 \sigma^{2}}}, \\
x, y & =0, \ldots, 2 \omega
\end{aligned}
$$

where $G, \sigma$ and $2 \omega$ are the gain factor, standard deviation, and template width, respectively. The flat field frames (blank images) were used for creating the unique the map of dust pattern of DSLR camera. The results of the mentioned method are depicted in Figure 5.

To prevent false detections, lens parameter-dependent characteristics of dust spots are also taken into consideration. Experimental results show that the proposed detection scheme can be used in identification of the source digital single lens reflex camera at low false positive rates, even under heavy compression and downsampling.

\subsection{Hot pixels pattern}

In modern CCD matrices, the size of phototransistors (sensor element - sensel) is nanometres, and the total number is measured in millions. Thus, the appearance of defective elements is natural. When they are in the "breakdown" state, they pass the full supply voltage to the output, in the image they remain points with the maximum amplitude of all the colour channels (white colour), and are called "hot pixel". When they are in the "breakage" state, then despite any bright incident light, they do not react and in the image they respectively make a point with zero amplitudes ("dead pixel").

Due to the specific perception and images themselves, the points with the maximum luminosity are the most visible and obvious for both human and computer vision algorithms.

The process of identifying the "hot pixel" can be described in the following way. As we have already established the appearance of a "hot pixel" does not depend on the image to be shot, it is not needed to remove them. The camera releases the shutter with the lens cap closed. Thus, all sensels in a good condition should give an black tone, and the desired one - the maximum value that is different from them. Completely to achieve value in one it is impossible in view of a self-tuning a sensor matrix power in the camera. For definiteness, let's select the shutter speed in $1 / 10 \mathrm{~s}$, as the middle of the range available to the device. For the independence of the experiment from further pre- and postprocessing of the device, images were taken in RAW format for Canon *.CR2.

An illustration of the process for the Canon EOS 40D (older in age and time in use) and Canon EOS D700 (newer) is shown in Figure 6. It is important to note that photo resistors, although designed to fix the image in the optical range, but because of the internal structure are able to register and radiation in other bands. To neutralize random fluctuations, it is necessary to average the result of fixation on a set of images. Averaging was carried out for a series of 30 frames of each cameras. This is clearly seen in the illustrations in Figure 6 (a), where the search process is performed for each image of set, it is stored and then everything is applied to the resulting averaged image. Figure 6 (d) depicts a case, when the images are averaged firstly, and then a search for mentioned points are performed.

Figure 6 shows that the camera Canon EOS 40D has reliable characteristic features in the form of points "hot pixel". This is confirmed by the experiment for each of the images in Figure 6 (b,c). The coordinates of all the points found on the series were sorted. Strongly visible steps correspond to the fact that there was a large number of points with such a coordinate.

In the case of the Canon EOS D700, there are only a few such steps, and they are not so long that they are hardly recognizable and difficult to formalize for automatic searching. This suggests that the new device should not actually have defects from the factory.

These sharp changes are an excellent illustration that the points of "hot pixel" are characteristic of each DSLR camera due to its individual operating conditions.

\subsection{Fixed pattern noise}

Due to unknown pattern of fixed pattern noise (FPN) of DSLR camera sensors all works in this field of science based on only consuming of this noise model.

Recently, similar to (Kurosawa, 1999), (Lukas, 2006) and (Chen, 2007a), (Chen, 2007) proposed a more reliable sensor noise-based source digital camera and camcorder identification method. Their method is based on the extraction of the unique photoresponse nonuniformity (PRNU) noise pattern which is caused by the impurities in silicon wafers and sensor imperfections. These imperfections affect the light sensitivity of each individual pixel and cause a fixed noise pattern. Similarly, (Khanna, 2007), (Swaminathan, 2007), and recently (Gloe, 2007) have ex- tended PRNU noise extraction methodology to source scanner identification where the imaging sensor is typically a 1-D linear array. The drawback of this approach is that it is very hard to synchronize the scanner noise pattern with the noise residue extracted from the scanned image. This is due to difficulty in controlling the document position during scanning. Therefore, aforementioned authors extracted statistical characteristics of PRNU noise and deployed machine learning methods to identify the scanner brand and model. It should be noted, that utilizing feature-based classifiers makes these methods less effective in individual source scanner identification. An example of the described method is depicted in Figure 7.

As this instance shows, FPN artefacts appear in digital images as color vertical strips. These strips are unique for each digital source.

\subsection{CFA traces}

The most practices method for detecting camera unique trace fo digital forensic task is connected with to color interpolation artefacts finding. Most state-of-the art digital cameras employ a single color filter array (CFA) rather than having different filters for each color band to generate color images. As a result of using a single CFA, each pixel is only represented by single color value and an interpolation operation has been performed to obtain the missing color values at each pixel operation. Therefore, demosaicing is a common processing technique that is central to operation of most digital cameras. Due to its proprietary design and implementation, the choice of CFA and demosaicing operation provides a unique opportunity to determine the source camera-model of a given digital image. 

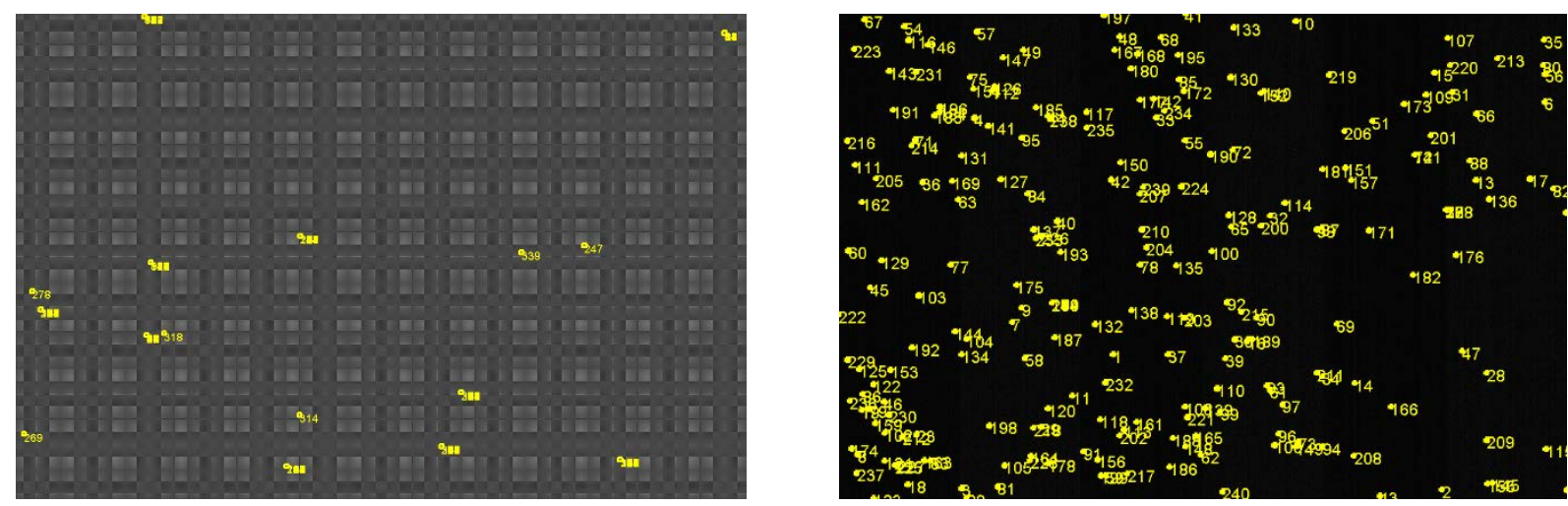

a)
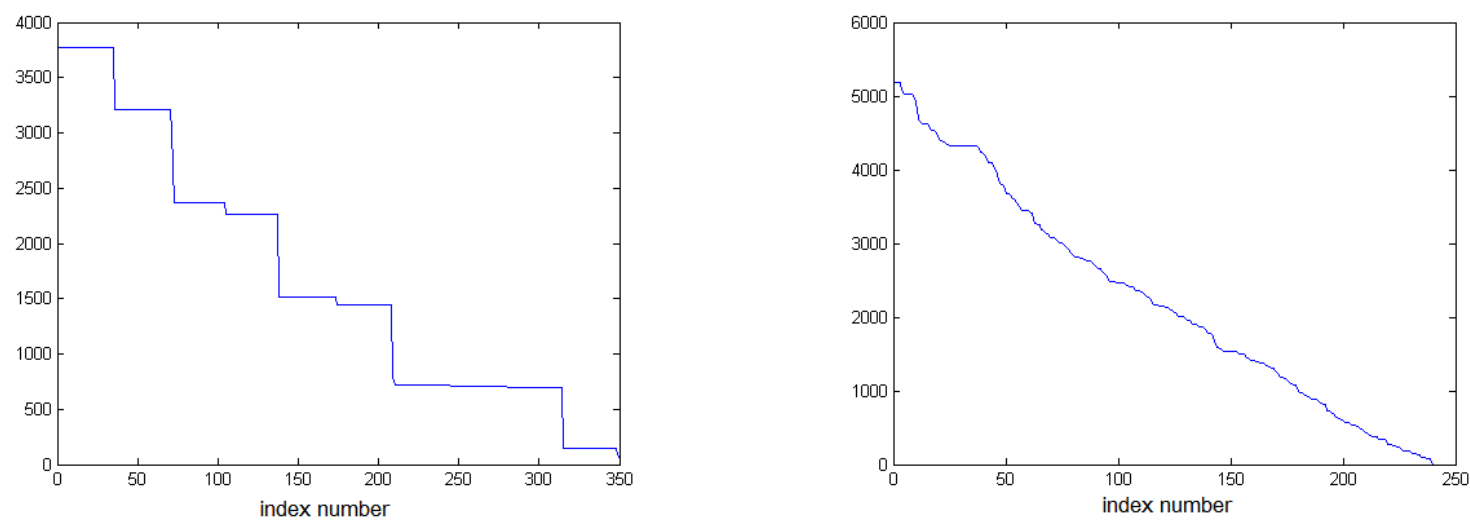

b)
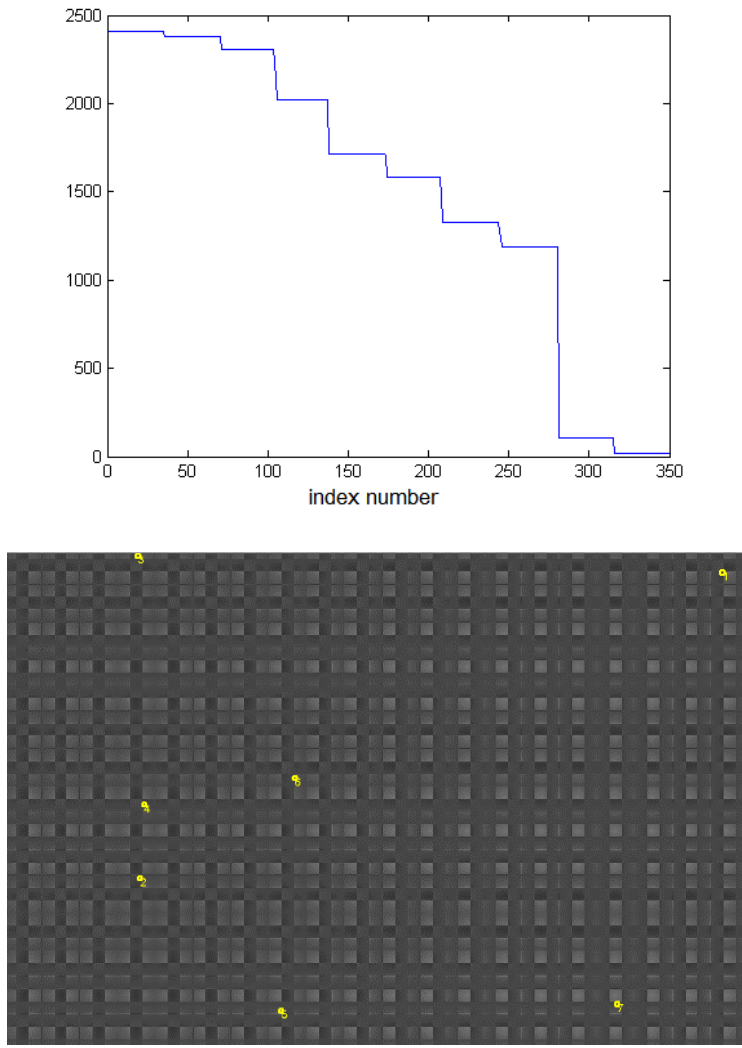

c)
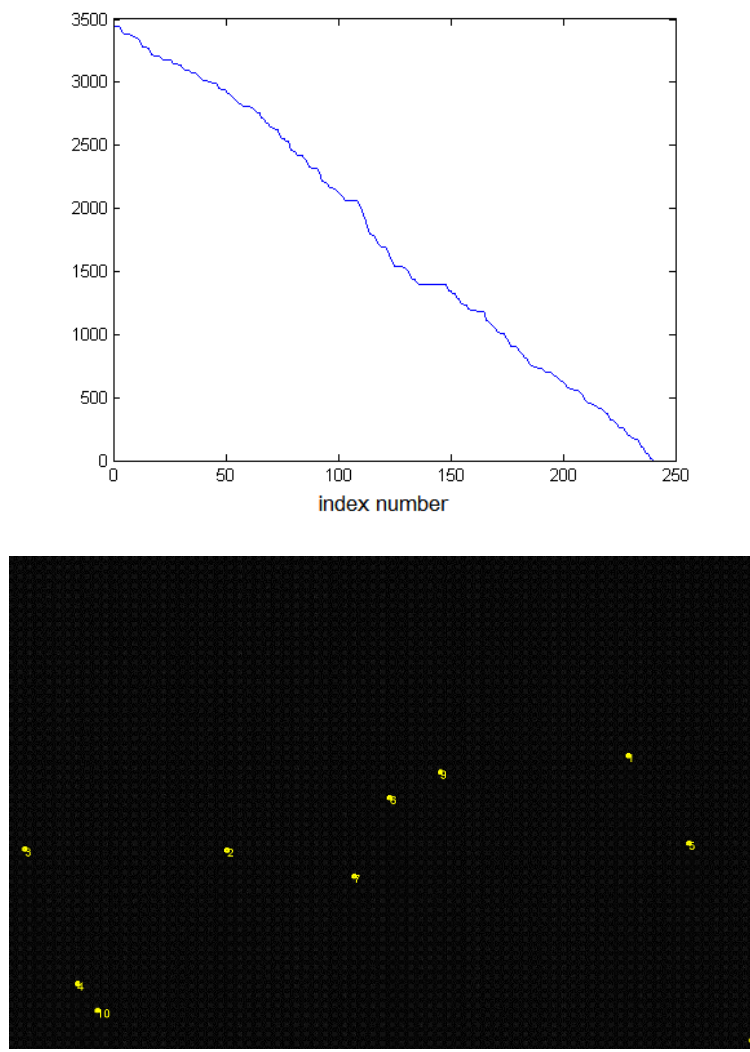

d)

Figure 6. The results of hot pixels pattern algorithm for Canon 40D (left column) and Canon 700D (right column): a) without averaging; b) the sorted $x$ coordinate of image; c) the sorted $y$ coordinate of image; d) the averaged image 


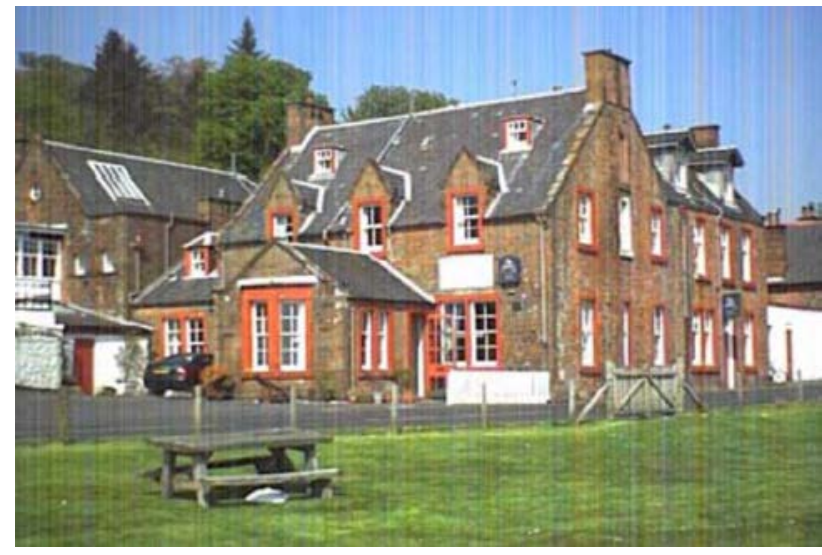

Figure 7. FPN traces on digital image

To detect and classify the traces of interpolation operation in images we rely on two methods. The first method is based on the use of Expectation-Maximization algorithm, which analyses the correlation of each pixel value to its neighbours (Popescu, 2005). The second method is based on analysing inter-pixel differences (Gallagher, 2005). Between the two methods, the former one can be also applied to less smooth parts of the image where the variation is not significant. On the other hand the latter is better suited for extremely smooth parts of the image where a simpler form of interpolation is deployed. In our analysis, we partition the image into blocks based on the level of smoothness decided using relative deviation of the variance in each block.

The Expectation/Maximization (EM) algorithm consists of two major steps: an expectation step, followed by a maximization step (Moon, 1996). The expectation is with respect to the unknown variables, using the current estimate of the parameters, and conditioned upon the observations. The maximization step then provides a new estimate of the parameters. These two steps are iterated until convergence. The EM algorithm generates two outputs. One is a two-dimensional data array, called probability map, in which each entry indicate the similarity of each image pixel to one of the two groups of samples, namely, the ones correlated to their neighbors and those ones that are not, in a selected kernel. The other output is the estimate of the weighting (interpolation) coefficients which designate the amount of contribution from each pixel in the interpolation kernel.

Since color filter arrays have more green color values, the red and blue color channels are more heavily interpolated. Therefore, we employed EM algorithm only on red channel; however, it can be trivially extended to other channels. Since no a priori information is assumed on the size of interpolation kernel probability maps and weighting coefficients are obtained for varying sizes of kernels.

Experimental results showed that the accuracy improves with the increasing kernel sizes. Therefore, in this paper, we assume $5 x 5$ interpolation kernel. Hence, the EM algorithm is used to estimate the weighting coefficients in $5 x 5$ neighbourhoods.

Low-order interpolation introduces periodicity in the variance of the second-order derivative of an interpolated signal, which can be used to determine the rate of interpolation (Gallagher, 2005). In this regard, the method first obtains the second-order derivative of each row and averages it over all rows.
If the image is interpolated, this pseudo-variance signal exhibits a periodicity. When observed in the frequency domain the locations of the peaks of the variance signal reveal the interpolation rate and the magnitude of the peaks deter- mine the interpolation method. We employed a similar methodology to characterize the interpolation rate and the method employed by a digital camera.

Most digital cameras encode and compress images in JPEG format. Due to $8 x 8$ block coding in JPEG, the DC (digital cosine) coefficients may also introduce peaks in the second-order derivative implying the presence of some form of interpolation operation at a rate of 8 . Therefore, in detecting the interpolation algorithm, the peaks due to JPEG compression have to be ignored. The variation in magnitude and indicates that there are differences in the deployed interpolation algorithm.

\section{CONCLUSIONS}

To sum up, solution of digital image forensics task could be based on unique noise pattern features. There are a few approaches to find these noise characteristics. The up-to-date methods of optical and sensor digital artefacts and noises were taken into consideration in this paper. The original algorithm of hot pixel detection was proposed.

Our future works will be connected with unique noise DSLR cameras analyses of features, choice unique of them and to try fixed pattern noise construction since we guess that this type of DSLR camera noise is the most complex and could be used for the aimed, which are mentioned in Section 1.

\section{ACKNOWLEDGEMENTS}

We would like to thank Yuriy Brjukhanov, Yuriy Lukashevich and Vladimir Volokhov from Yaroslavl State University, who helped us with the equipment to collect database of testing images and produced us the opportunity to realize our possibilities. This work was supported by RFBR Grant 15 0899639-a.

\section{REFERENCES}

Buades, A., 2005. A non-local algorithm for image denoising. IEEE Computer Society Conference on Computer Vision and Pattern Recognition 2, pp. 60-65.

Chen, M., 2007a. Digital imaging sensor identification (further study), Proc. SPIE Security, Steganography, Watermarking of Multimedia Contents IX, Vol. 6505, p. 65050.

Chen, M., 2007b. Source digital camcorder identification using sensor photo response non-uniformity, Proc. SPIE Security, Steganography, Watermarking of Multimedia Contents IX, Vol. 6505.G, p. 65051, 2007.

Choi, K., 2006. Source camera identification using footprints from lens aberration, Digital photography II. In Proc. of the SPIE, Vol. 6069, pp. 172-179.

Dirik, A., 2007. Source camera identification based on sensor dust characteristics. In Proc. IEEE Workshop Signal Processing Applications for Public Security and Forensics, pp. 1-6.

Gallagher, A., 2005. Detection of linear and cubic interpolation in JPEG compressed images. In: Proceedings of CRV, pp. 65-73. 
Gloe, T. 2007. Forensics for flatbed scanners. In Proc. Security, Steganography, and Watermarking of Multimedia Contents IX, Vol. 6505, p. 65051.

Kijewski, M., 1987. The noise power spectrum of ct images. Physics, Medicine, Biology, 32(5), pp. 565-575.

Reiter, M., 2006. Denoising of computer tomography images using multiresolution based methods. European Conference on Non-Destructive Testing poster.

Thinh, D., 2012. An optimal weight method for ct image denoising. Journal of Electronoc Science and Technology, 10(2), pp. 124-129.

Strozhilova, M., 2012.Two approaches for noise filtering in 3d medical ct-images. In: 22-th International Conference on Computer Graphics GraphiCon'2012, Moscow, Russia, pp. 6872.

Khanna, N., 2007. Scanner identification using sensor pattern noise, Proc. SPIE Security, Steganography, Watermarking of Multimedia Contents IX, Vol. 6505, p. 65051.

Kharrazi, M., 2004. Blind source camera identification. In: IEEE international conference on image processing, Vol. 1. pp. 709712 .

Kurosawa K., 1999. CCD Fingerprint method - Identification of a video camera from videotaped images, in Procc. ICIP, Kobe, Japan, pp. 537-540.

Long, Y., 2006. Image based source camera identification using demosaicking. In: IEEE 8th workshop on multimedia signal processing, Victoria, BC. pp. 419-424.

Lukas, J., 2006. Digital camera identification from sensor noise. IEEE Trans. Inf. Forensics Security, Vol. 1, no. 2, pp. 205-214.

Moon, T., 1996. The expectation maximization algorithm. IEEE Signal Processing Magazine, pp.47-60.

Popescu, A., 2005. Exposing digital forgeries in colour filter array interpolated images. IEEE Transactions on Signal Processing 2005, 53(10), pp. 3948-3959.

Swaminathan, A., 2007. Non intrusive forensic analysis of visual sensors using output images. IEEE Transactions of Information Forensics and Security, 2(1), pp. 91-106. 